\title{
URINARY GLYCOSAMINOGLYCAN EXCRETION DURING THE MENSTRUAL CYCLE IN NORMAL YOUNG WOMEN
}

\author{
MARCUS V. O. MAROCLO, SÁVIO D. PEREIRA, FRANCISCO J. B. SAMPAIO \\ AND LUIZ E. M. CARDOSO* \\ From the Urogenital Research Unit, State University of Rio de Janeiro, Rio de Janeiro, Brazil
}

\section{ABSTRACT}

Purpose: We investigated whether the menstrual cycle affects urinary glycosaminoglycan (GAG) excretion in normal young women.

Materials and Methods: Urine samples from 10 healthy women 19 to 21 years old were collected daily during the whole menstrual cycle. Concentration of total urinary GAG was assessed as $\mu \mathrm{g}$ hexuronic acid per mg creatinine. Proportions of sulfated GAG species were determined by agarose gel electrophoresis.

Results: Mean excretion values \pm SD for period days 4 to 13 and 15 to 28 of the cycle were significantly different $(0.445 \pm 0.041$ vs $0.356 \pm 0.035 \mu \mathrm{g} / \mathrm{mg}, \mathrm{p}<0.001)$. Correlation between values for the first and second halves of the cycle showed that this difference was consistent irrespective of individual variations in GAG excretion $(r=0.9757, p<0.001)$. Proportions of urinary sulfated GAG did not change during the cycle.

Conclusions: Excretion of total urinary GAG during the normal menstrual cycle of young women has a biphasic pattern with significantly higher values occurring in the first half of the cycle. This variation implies modulation by estrogens and consequently it should be considered when comparing the GAG concentration in urine samples from women of childbearing age.

KEY WoRDs: bladder; cystitis, interstitial; glycosaminoglycans; menstrual cycle; urine

Proteoglycans and their glycosaminoglycan (GAG) side chains are complex extracellular matrix molecules that, in addition to a structural role, are implicated in a wide range of normal and pathological cellular processes. In comparison to other components of the extracellular matrix, proteoglycans have a high turnover rate. Their degradation usually involves the extracellular action of matrix metalloproteinases, of which cleavage products as well as smaller proteoglycan monomers are subsequently incorporated by cells and further degraded. ${ }^{1}$ Proteoglycan fragments and free hyaluronan filaments may also permeate through the interstitium and reach the blood stream, from which they are rapidly taken up by liver cells and metabolized. ${ }^{2}$ Indeed, if radiolabeled proteoglycan or GAG is injected intravenously in experimental animals, most recovered radioactivity is in the liver, ${ }^{2}$ whereas in urine it will be found as low molecular weight metabolites. ${ }^{3}$ As a consequence of these metabolic pathways, the serum concentration of GAG chains is itself relatively low compared with the amount that is normally eliminated in urine. Actually, renal filtration of GAG is small and most excreted chains are thought to derive from the normal proteoglycan turnover that occurs locally in the glomerulus and urinary tract. ${ }^{4}$

Urinary excretion of GAG may be abnormally increased in a number of conditions that affect proteoglycan turnover, such as cancer ${ }^{5}$ and diabetes. ${ }^{6}$ In addition, it has been suggested that the determination of urinary GAG excretion in many of these conditions may be useful for diagnosis and/or followup.

There is now experimental evidence indicating that female

Submitted for publication August 27, 2004.

Supported by grants from the National Council of Scientific and Technological Development (CNPq), Foundation for Research Support of Rio de Janeiro (FAPERJ) and Coordination for Improvement of Post-Graduated Students (CAPES), Brazil.

* Correspondence: Urogenital Research Unit-UERJ, Av. 28 de Setembro, 87-fundos-FCM-térreo, 20551-030, Rio de Janeiro, RJ, Brazil (FAX: + 55 21- 2587-6121; e-mail: lemc@uerj.br). sex hormones modulate extracellular matrix remodeling and proteoglycan turnover in vivo ${ }^{7}$ and in vitro. ${ }^{8}$ Therefore, normal menstruation, which is accompanied by marked changes in the serum levels of such hormones, could modulate proteoglycan metabolism. However, few groups have analyzed urinary GAG excretion during the menstrual cycle ${ }^{9-11}$ and their results are conflicting for various technical reasons. Such data would be particularly important for investigations of urinary GAG excretion in women of childbearing age. Thus, if variations in GAG excretion exist, urine samples to be compared would have to be collected at known days of the menstrual cycle, otherwise erroneous or misleading results might be obtained. Indeed, the lack of consensus regarding GAG excretion in interstitial cystitis, ${ }^{11-13}$ an often debilitating disease that affects mostly women, may be due to the use of inadequate samples.

In this study we addressed this issue by investigating urinary GAG excretion during the normal menstrual cycle of young women. We used well established analytical methods and daily samples from a homogeneous and controlled group of urine donors.

\section{MATERIALS AND METHODS}

Urine samples. Urine samples were voluntarily donated by 10 healthy white female medical students. All donors met study exclusion criteria (see Appendix). Donor age was 19 to 21 years. Early in the morning each student collected 1 urine specimen for each day of the menstrual cycle, starting on the first day of bleeding and ending on the first day of the next cycle. Cycle duration was 24 to 29 days (mean \pm SD $27.30 \pm$ 1.56). Immediately after collection urine samples were frozen without adding preservatives and stored at $-20 \mathrm{C}$ until further analyses.

Isolation of total urinary GAG. GAGs were isolated separately from each daily urine sample. Thus, samples were thawed at the laboratory, filtered on standard filter paper 
(No. 40, Whatman International, Bath, United Kingdom) and density was adjusted to less than 1.020 by adding distilled water. Cetylpyridinium chloride (152 $\mu \mathrm{l} 10 \%)$ was added to $10 \mathrm{ml}$ diluted urine. After vortexing the mixture was left at room temperature for 24 hours. Precipitates were collected by centrifugation at $3,000 \times$ gravity for 30 minutes on a standard clinical bench centrifuge. The pellet was washed with $15 \mathrm{ml} 0.05 \%$ cetylpyridinium chloride and dissolved in 2 $\mathrm{ml} 2 \mathrm{M} \mathrm{NaCl} /$ absolute ethanol (100:15 volume per volume). Three volumes of absolute ethanol were then added and the mixture was kept at $4 \mathrm{C}$ for 24 hours. Precipitates were collected by centrifugation at $3,000 \times$ gravity for 30 minutes. The pellet was washed twice with $10 \mathrm{ml} 70 \%$ ethanol and once with $10 \mathrm{ml}$ absolute ethanol. The final pellet, which represented total urinary GAG, was dried at $60 \mathrm{C}$ for 30 minutes, dissolved in $200 \mu \mathrm{l}$ distilled water and kept at $-20 \mathrm{C}$ until further analyses. The recovery of this method was in excess of $95 \%$, as determined by the addition of known amounts of GAG standards to urine samples.

Quantitation of urinary GAG. The concentration of total GAG in each daily urine sample was determined as hexuronic acid by the carbazole method ${ }^{14}$ using glucuronolactone (Sigma Chemical Co., St. Louis, Missouri) as the standard. These results were then normalized to the creatinine concentration of each urine sample, so that the urinary GAG concentration is expressed as $\mu \mathrm{g}$ hexuronic acid per mg creatinine.

Relative concentration of sulfated GAG species. The relative concentration of urinary sulfated GAG species was determined by electrophoresis on $0.5 \%$ agarose (type I-B, Sigma Chemical Co.). ${ }^{15}$ Toluidine blue stained gels were then digitized and the density of stained bands was measured with Scion Image, version 4.0.2 software (Scion Corp., Frederick, Maryland). The relative content of individual GAG species is expressed as a percent of total sulfated GAG. Identification of GAG species was based on specific degradative treatments, as previously described. ${ }^{15}$

Other analytical methods. Urine samples were tested with dipsticks (Multistix 10G, Bayer, Tarrytown, New York) for leukocytes, erythrocytes, $\mathrm{pH}$, density, total protein and glucose. Urinary creatinine was determined by an automated enzyme assay (Sera-Pack, Bayer, Tarrytown, New York).

Statistical analysis. Before statistical analysis of urinary GAG excretion menstrual cycles were normalized to 28 days using the formula, $\mathrm{Dn}=(\mathrm{Dc} \times 28) / \mathrm{Dd}$, where $\mathrm{Dn}$ represents normalized day, which was rounded to the nearest integer, Dc represents the day number in the nonnormalized cycle and Dd represents the number of days in the nonnormalized cycle. If consecutive day numbers in the nonnormalized cycle (Dc) yielded the same integer (Dn), the GAG excretion value assigned to this normalized day number was the mean of the corresponding day numbers in the nonnormalized cycle.

To evaluate the excretion pattern of urinary GAG we arbitrarily divided the normalized menstrual cycles into 2 or 3 phases, which were named pre-ovulatory and post-ovulatory or pre-ovulatory, ovulatory and post-ovulatory, respectively. The precise days that delimited these phases are indicated. Differences in the excretion of total GAG were evaluated by 1-way ANOVA, whereas proportions of sulfated GAG were compared using the Kruskal-Wallis test. Correlation analysis involved the calculation of the correlation coefficient, followed by a t test for this coefficient. Statistical procedures were performed as previously described ${ }^{7}$ with statistical significance considered at $\mathrm{p}<0.05$.

\section{RESULTS}

Excretion of total urinary GAG in normal young women presented a biphasic pattern with higher concentration values during the first half of the menstrual cycle (fig. 1). To ascertain whether this difference was statistically significant
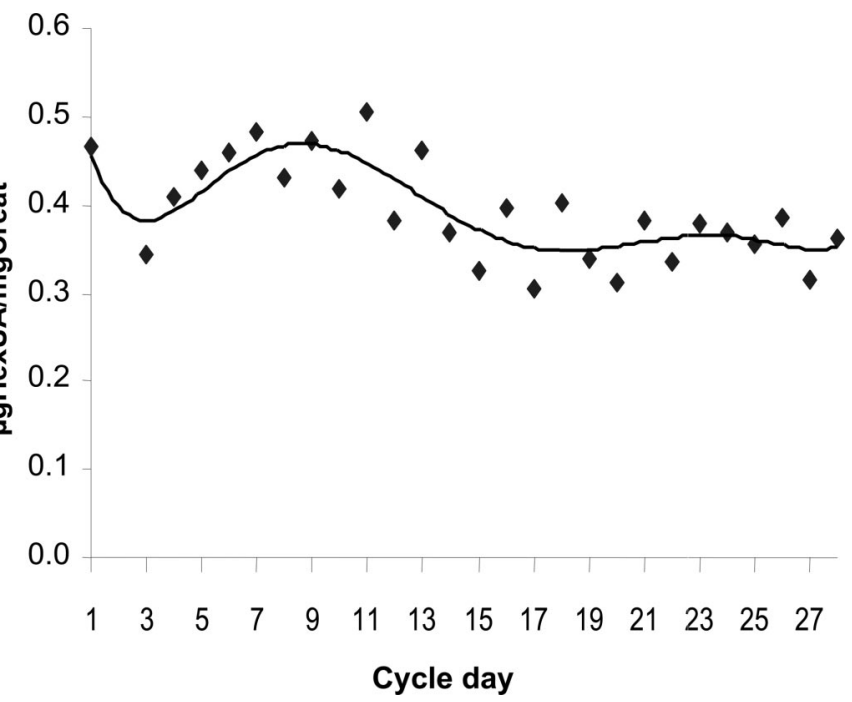

FIG. 1. Daily excretion of total urinary GAGs during normal menstrual cycle of young women. Regression line was drawn using nonlinear polynomial regression. Diamonds represent mean GAG in 10 individuals for given day of normalized cycle. $\mu \mathrm{gHexUa} / \mathrm{mgCreat}, \mu \mathrm{g}$ hexaluronic acid per mg creatinine.

we used mean daily values to calculate mean GAG excretion for the pre-ovulatory (days 4 to 13 in 10 preparations) and post-ovulatory (days 15 to 28 in 14 preparations) periods (figs. 1 and 2). Comparison of these 2 means showed that average urinary GAG excretion in the pre-ovulatory phase was $25.0 \%$ higher than that in the post-ovulatory phase $(0.445 \pm$ $0.041 \mathrm{vs} 0.356 \pm 0.035 \mu \mathrm{g}$ hexuronic acid per mg creatinine, $\mathrm{p}<0.001$ ).

Figure 1 shows that there was noticeable individual and daily variation in GAG excretion, so that the difference that we found between pre-ovulatory and post-ovulatory mean GAG values might not have been valid for all baseline values. Thus, to determine whether this difference in GAG excretion was consistent among individuals, correlation analysis was done between the pre-ovulatory and post-ovulatory phases (fig. 3). The results showed a strong and significant correla-

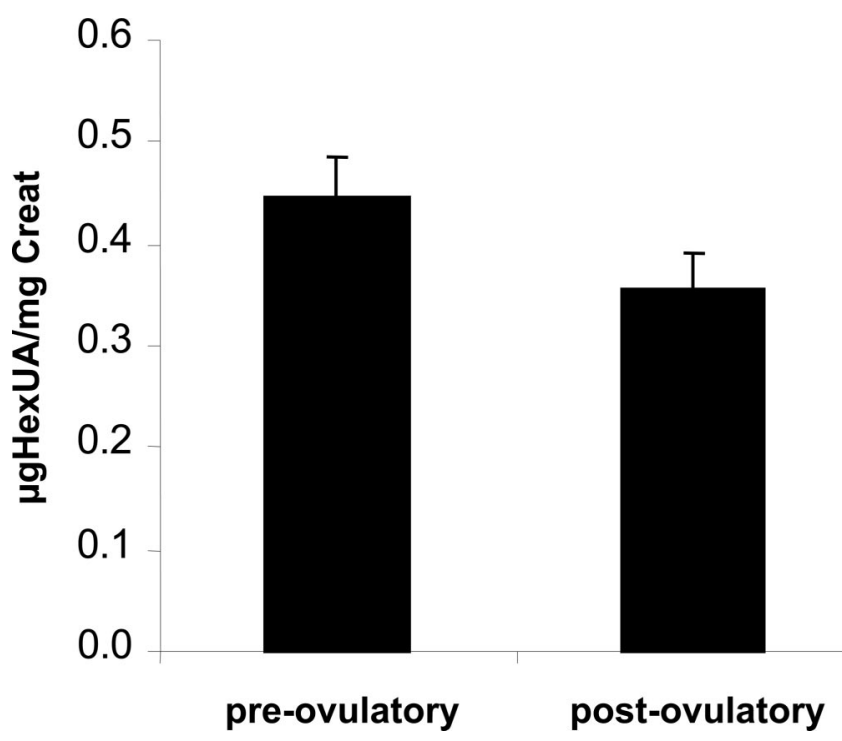

FIG. 2. Mean excretion of total urinary GAG in menstrual cycle pre-ovulatory and post-ovulatory phases. Bars represent daily average excretion between days 4 and 13 (pre-ovulatory in 10 preparations), and 15 and 28 (post-ovulatory in 14 preparations) \pm SD (fig. 1). $\mu g$ HexUa/mg Creat, $\mu \mathrm{g}$ hexaluronic acid per mg creatinine. Means were significantly different (1-way ANOVA $\mathrm{p}<0.001$ ). 


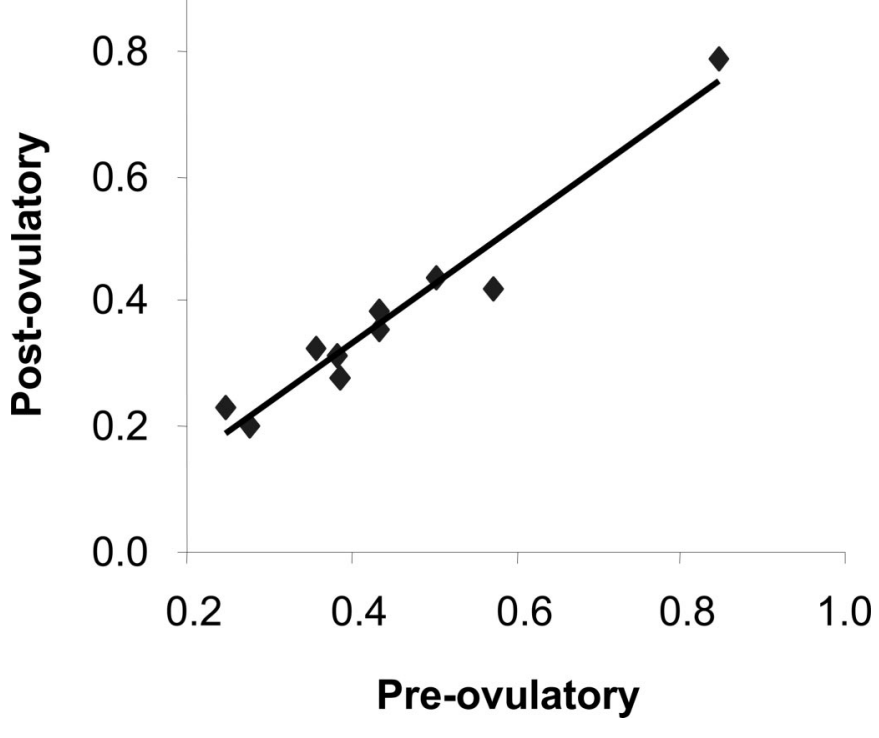

FIG. 3. Correlation analysis between pre-ovulatory and postovulatory daily excretion of urinary GAG. For each individual mean daily urinary GAG (diamonds) for menstrual cycle days 4 to 13 (pre-ovulatory) and 15 to 28 (post-ovulatory) were plotted and correlation analysis was performed for these value pairs $(r=0.9757$, $\mathrm{p}<0.001)$.

tion $(\mathrm{r}=0.9757, \mathrm{p}<0.001)$, which implied that GAG excretion was consistently higher in the pre-ovulatory phase of the menstrual cycle and it was independent of individual or daily variations in baseline GAG excretion.

The biphasic pattern of total GAG excretion during the menstrual cycle was apparent in our analysis of daily concentration values (fig. 1), so that for statistical comparison we divided the cycle into 2 phases only. However, for analysis of the relative concentration of urinary sulfated GAG species there was no prior clue indicating a specific excretion pattern, and so we divided the menstrual cycle into 3 phases to increase resolution. In all 3 phases (pre-ovulatory days 6 to 11 , ovulatory days 12 to 17 and post-ovulatory days 18 to 28 ) heparan sulfate was the prevailing GAG, representing $55.9 \%$ to $60.1 \%$ of total sulfated GAG (fig. 4). The results also showed that the proportions of the 3 GAG species were almost identical among the 3 phases.

\section{DISCUSSION}

Previous reports including data on urinary GAG excretion during the normal menstrual cycle differ appreciably with regard to their results. For example, Erickson et al did not find consistent variations during the normal cycle. ${ }^{11}$ However, their group of urine donors was not homogeneous. Also, the assay method used, which was based on dye binding, can be affected by GAG composition and it is more prone to interference by urinary metabolites. ${ }^{16}$ In another report, which used a small number of urine donors (4) who collected samples at a 5-day interval, similar results were obtained. ${ }^{9}$ In contrast, significant variation in the urinary GAG concentration during ovulation was reported by Carranco et al, who suggested that this could be an efficient method for determining this event. ${ }^{10}$ However, in the latter report a dye binding method for assaying GAG was also used and GAG excretion values were not normalized to urinary creatinine. Therefore, the daily urinary GAG concentration could have been affected by factors such as water intake, environmental temperature and dehydration. Thus, because of inconsistent approaches in relation to analytical methods, and the selection of samples and donors, a consensus is still lacking with regard to the excretion pattern of urinary GAG during the normal menstrual cycle.

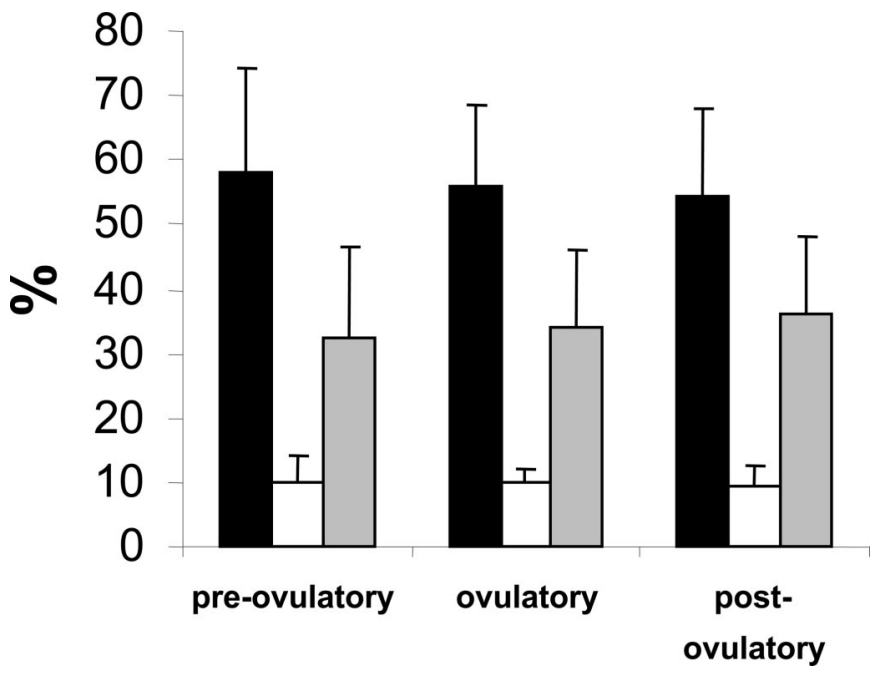

FIG. 4. Relative concentration of sulfated urinary GAGs in different menstrual cycle phases. For each individual total GAGs isolated from daily urine samples were pooled according to phase (preovulatory days 6 to 11 , ovulatory days 12 to 17 and post-ovulatory days 18 to 28 ) and submitted to agarose gel electrophoresis. Relative concentrations of heparan sulfate (black bars), dermatan sulfate (open bars) and chondroitin sulfate (gray bars) are expressed as percent of total sulfated GAG. Bars represent mean \pm SD of 10 individuals. Proportion of each GAG was compared among 3 phases and no significant differences were found (Kruskal-Wallis test).

In the current study urine samples were obtained on a daily basis from a highly homogeneous group of donors. We then isolated total GAG from these samples, thereby, eliminating other metabolites and we used a hexuronic acid assay to determine the GAG concentration. Unlike dye binding methods, this assay is not appreciably affected by charge density or the presence of different GAG species. ${ }^{14}$ Our results showed a significant increase in total urinary GAG excretion in the first half of the cycle, which paralleled the normal increase in serum estrogen levels that occurs at this phase. In general, estrogen inhibits the synthesis of extracellular matrix molecules by many mesenchymal cell types, such as vascular smooth muscle cells. ${ }^{17}$ Such inhibition would shift normal proteoglycan turnover toward degradation, which could explain the increase in GAG urinary excretion that we found in the first half of the cycle.

We did not observe significant variation in the relative concentration of sulfated GAG during the different phases of the cycle. On the other hand, our results indicate that heparan sulfate was the prevailing urinary GAG during the whole cycle. Because heparan sulfate is the most abundant GAG in the glomerulus, ${ }^{18}$ our findings support the hypothesis that renal structures are one of the main sources of urinary GAG. ${ }^{4}$

The pathogenesis of interstitial cystitis is usually related to alterations in the GAG urothelial layer, which would allow the permeation of irritant urinary components into the vesical wall. ${ }^{12}$ Several reports have shown abnormal urinary GAG excretion in patients with interstitial cystitis, although the results are conflicting. Accordingly, urinary GAG levels in female patients may be decreased ${ }^{12}$ unaltered $^{11}$ or significantly increased. ${ }^{13}$ In these reports controls usually consisted of urine samples from healthy women of childbearing age. However, the dates of the menstrual cycle in which these control samples were collected were not provided. Since our results indicate that urinary GAG excretion during the normal menstrual cycle has a significant and consistent variation, studies evaluating GAG excretion in women could lead to misleading or erroneous results if comparisons were made among samples taken from different phases of the cycle. This may be indeed the reason underlying the inconsistent results in previously published reports, as described. 
Many therapeutic approaches have been used to treat the pelvic pain associated with interstitial cystitis and the oral or vesical administration of solutions of GAG or GAG-like glycans is usually considered to be effective with regard to symptom relief. ${ }^{19}$ The beneficial effects of this treatment are thought to derive from the local deposition of glycan chains, thereby, repairing and/or protecting a putatively damaged urothelial surface. Therefore, the decrease in GAG excretion in the second half of the menstrual cycle, as reported, may explain the findings of Lentz et al, who observed that pelvic pain in patients with interstitial cystitis is exacerbated at the end of the menstrual cycle. ${ }^{20}$

\section{CONCLUSIONS}

Excretion of total urinary GAG during the normal menstrual cycle of young women has a biphasic pattern with significantly higher values occurring in the first half of the cycle. This variation implies modulation by estrogens and consequently it should be considered when comparing the GAG concentration in urine samples from women of childbearing age.

APPENDIX: EXCLUSION CRITERIA FOR ENROLLING FEMALE VOLUNTEERS IN THE STUDY

1) Use of oral contraceptives

2) History of bladder or renal stones

3) Body mass index outside the 20 to 25 range

4) Symptoms of urinary infection shortly before or during the menstrual cycle under study

5) Irregular menstrual cycles

6) Diabetes mellitus

7) Abnormal result on dipstick test

\section{REFERENCES}

1. Cawston, T. E.: Metalloproteinase inhibitors and the prevention of connective tissue breakdown. Pharmacol Ther, 70: 163, 1996

2. Masuda, H., Takakura, Y. and Hashida, M.: Pharmacokinetics and disposition characteristics of recombinant decorin after intravenous injection into mice. Biochim Biophys Acta, 1426: 420,1999

3. Brown, T. J., Alcorn, D. and Fraser, J. R.: Absorption of hyaluronan applied to the surface of intact skin. J Invest Dermatol, 113: 740,1999

4. Lee, E. Y., Kim, S. H., Whang, S. K., Hwang, K. Y., Yang, J. O. and Hong, S. Y.: Isolation, identification, and quantitation of urinary glycosaminoglycans. Am J Nephrol, 23: 152, 2003

5. Lokeshwar, V. B., Obek, C., Pham, H. T., Wei, D., Young, M. J., Duncan, R. C. et al: Urinary hyaluronic acid and hyaluronidase: markers for bladder cancer detection and evaluation of grade. J Urol, 163: 348, 2000
6. Juretic, D., Krajnovic, V. and Lukac-Bajalo, J.: Altered distribution of urinary glycosaminoglycans in diabetic subjects. Acta Diabetol, 39: 123, 2002

7. Cabral, C. A. P., Sampaio, F. J. B. and Cardoso, L. E. M.: Analysis of the modifications in the composition of bladder glycosaminoglycan and collagen as a consequence of changes in sex hormones associated with puberty or oophorectomy in female rats. J Urol, 170: 2512, 2003

8. Tanaka, K., Nakamura, T., Takagaki, K., Funahashi, M., Saito, Y. and Endo, M.: Regulation of hyaluronate metabolism by progesterone in cultured fibroblasts from the human uterine cervix. FEBS Lett, 402: 223, 1997

9. Ledger, W. J., Thompson, G. R. and Grobelny, S. L.: Effects of the menstrual cycle, pregnancy, hysterectomy and other operative procedures upon urinary mucopolysaccharide excretion: a preliminary report. Amer J Obstet Gynecol, 110: 494, 1971

10. Carranco, A., Reyes, R., Huacuja, L., Guzman, A. and Delgado, N. M.: Human urinary glycosaminoglycans as accurate method for ovulation detection. Int J Fertil, 37: 209, 1992

11. Erickson, D. R., Ordille, S., Martin, A. and Bhavanandan, V.P.: Urinary chondroitin sulfates, heparan sulfate and total sulfated glycosaminoglycans in interstitial cystitis. J Urol, 157: 61, 1997

12. Parsons, C. L. and Hurst, R. E.: Decreased urinary uronic acid levels in individuals with interstitial cystitis. J Urol, 143: 690, 1990

13. Wei, D. C., Politano, V. A., Selzer, M. G. and Lokeshwar, V. B.: The association of elevated urinary total to sulfated glycosaminoglycan ratio and high molecular mass hyaluronic acid with interstitial cystitis. J Urol, 163: 1577, 2000

14. Taylor, K. A. and Buchanan-Smith, J. G.: A colorimetric method for the quantitation of uronic acids and a specific assay for galacturonic acid. Anal Biochem, 201: 190, 1992

15. Cardoso, L. E., Erlich, R. B., Rudge, M. C., PeraColi, J. C. and Mourao, P. A.: A comparative analysis of the glycosaminoglycans from human umbilical arteries in normal subjects and in pathological conditions affecting pregnancy. Lab Invest, 67: 588,1992

16. Farndale, R. W., Buttle, D. J. and Barrett, A. J.: Improved quantitation and discrimination of sulphated glycosaminoglycans by use of dimethylmethylene blue. Biochim Biophys Acta, 883: 173,1986

17. Barchiesi, F., Jackson, E. K., Gillespie, D. G., Zacharia, L. C., Fingerle, J. and Dubey, R. K.: Methoxyestradiols mediate estradiol-induced antimitogenesis in human aortic SMCs. Hypertension, 39: 874, 2002

18. Michelacci, Y. M., Cadaval, R. A., Rovigatti, R. M. and Kohlman, O.: Renal and urinary glycosaminoglycans in an experimental model of chronic renal failure in rats. Exp Nephrol, 9: 40, 2001

19. Dell, J. R. and Parsons, C. L.: Multimodal therapy for interstitial cystitis. J Reprod Med, 49: 243, 2004

20. Lentz, G. M., Bavendam, T., Stenchever, M. A., Miller, J. L. and Smalldridge, J.: Hormonal manipulation in women with chronic, cyclic irritable bladder symptoms and pelvic pain. Am J Obstet Gynecol, 186: 1268, 2002 